Global Journal of Pure and Applied Mathematics.

ISSN 0973-1768 Volume 11, Number 2 (2015), pp. 899-908

(C) Research India Publications

https://dx.doi.org/10.37622/GJPAM/11.2.2015.899-908

\title{
On The Probability That An Element of Metacyclic 2-Group of Positive Type Fixes A Set and Its Generalized Conjugacy Class Graph
}

\author{
Mustafa Anis El-Sanfaz ${ }^{a}$ and Nor Haniza Sarmin ${ }^{b}, *$ \\ ${ }^{a, b}$ Department of Mathematical Sciences, Faculty of Science, Universiti Teknologi \\ Malaysia, 81310 UTM Johor Bahru, Johor, Malaysia \\ ${ }^{a}$ Department of Mathematics, Faculty of Science, University of Benghazi. Benghazi,
}

Libya

\begin{abstract}
The probability that an element of a group fixes a set is considered as one of the extensions of commutativity degree that can be obtained by some group actions on a set. We denote $G$ as a metacyclic 2-group of positive type of nilpotency of class at least three and $\Omega$ as the set of all subsets of all commuting elements of $G$ of size two in the form of $(a, b)$, where $a$ and $b$ commute and each of order two. In this paper, we compute the probability that an element of $G$ fixes a set in which $G$ acts regularly on $\Omega$. Then the results are applied to graph theory, more precisely to generalized conjugacy class graph.
\end{abstract}

\section{Introduction}

Throughout this paper, $\Gamma$ denotes a simple undirected graph and $G$ denotes a finite non-abelian group. In the following, the definition of the commutativity degree is stated.

\section{Definition 1.1}

[1] Let $G$ be a finite non-abelian group. Suppose that $x$ and $y$ are two random elements of $G$. The probability that two random elements commute (also known as the commutativity degree) is given as follows.

$$
P(G)=\frac{|\{(x, y) \in G \times G: x y=y x\}|}{|G|^{2}} .
$$


The first investigation of the commutativity degree of symmetric groups was done in 1968 by Erdos and Turan [2]. Few years later, Gustafson [3] and MacHale [4] found an upper bound for the commutativity degree of all finite non-abelian groups in which $P(G) \leq \frac{5}{8}$. The concept of the commutativity degree has been generalized and extended by several authors. In 1975, a new concept was introduced by Sherman [5], namely the probability of an automorphism of a finite group fixes an arbitrary element in the group. The definition of this probability is given as follows:

\section{Definition 1.2}

[5] Let $G$ be a group. Let $X$ be a non-empty set of $G$ ( $G$ is a group of permutations of $X$ ). Then the probability of an automorphism of a group fixes a random element from $X$ is defined as follows:

$$
P_{G}(X)=\frac{|\{(g, x) \mid g x=x, \forall g \in G, x \in X\}|}{|G||X|} .
$$

In 2011, Moghaddam [6] explored Sherman's definition and introduced a new probability, which is called the probability of an automorphism fixes a subgroup element of a finite group, the probability is stated as follows:

$$
P_{A_{G}}(H, G)=\frac{\left\{(\alpha, h) \mid h^{\alpha}: h \in H, \alpha \in A_{G}\right\} \mid}{|H||G|},
$$

where $A_{G}$ is the group of automorphisms of a group $G$.It is clear that if $H=G$, then $P_{A_{G}}(G, G)=P_{A_{G}}$.

Recently, Omer et al [7] extended the work in [5] by defining the probability that a group element fixes a set. In this paper, the probability that a group element fixes a set is found for metacyclic 2-groups of positive type of nilpotency class at least three.

Next, we state some basic concepts in group theory that are needed in this paper.

\section{Definition 1.3}

[8] Suppose $G$ is a finite group that acts on a set $S$. A group $G$ acts regularly on $S$ if the action is transitive and $\operatorname{Stab}_{G}(s)=1$ for all $s \in S$. Also, the action is regular if for $s_{1}$ and $s_{2}$ belong to $S$, there exists an element $g \in G$ such that $g s_{1}=s_{2}$.

\section{Definition 1.4}

[9] Let $G$ acts on a set $S$ and $x \in S$. The orbit of $x$ denoted by $\operatorname{cl}(x)$ is the sub set $\operatorname{cl}(x)=\{g x: g \in G\} \subseteq S$.

Next, we provide some concepts related to metacyclic $p$-groups. Throughout this paper, $p$ denotes a prime number. 


\section{Definition 1.5}

[10] A group $G$ is called a metacyclic if it has a cyclic normal subgroup $H$ such that the quotient group $G / H$ is also cyclic.

In 1973, King [11] gave the presentation of metacyclic $p$-groups, as given in the follows:

$$
G \cong\left\langle a, b: a^{p^{\alpha_{1}}}=1, b^{p^{\alpha_{2}}}=a^{n},[b, a]=a^{m}\right\rangle, \text { where } \alpha_{1}, \alpha_{2} \geq 0, m>0, n \leq p^{\alpha_{1}}, p^{\alpha_{1}} \mid n(m-1) .
$$

In 2005, Beuerle [12] separated the classification into two parts, namely for the non-abelian metacyclic $p$-groups of class two and class at least three. Based on [12], the metacyclic $p$ - groups of nilpotency class two are then partitioned into two families of non-isomorphic $p$-groups stated as follows:

$$
\begin{aligned}
& G \cong\left\langle a, b: a^{p^{\alpha}}=1, b^{p^{\beta}}=1,[a, b]=a^{p^{\alpha-\gamma}}\right\rangle, \text { where } \alpha, \beta, \gamma \in \square, \alpha \geq 2 \gamma \text { and } \beta \geq \gamma \geq 1 . \\
& G \cong Q_{8} .
\end{aligned}
$$

Meanwhile, the metacyclic $p$-groups of nilpotency class of at least three ( $p$ is an odd prime) are partitioned into the following groups:

$$
\begin{aligned}
G \cong & \left\langle a, b: a^{p^{\alpha}}=1, b^{p^{\beta}}=1,[b, a]=a^{p^{\alpha-\gamma}}\right\rangle, \text { where } \alpha, \beta, \gamma \in \square, \alpha-1 \gamma<2 \text { and } \alpha \leq \beta . \\
G \cong & \left\langle a, b: a^{p^{\alpha}}=1, b^{p^{\beta}}=a^{p^{\alpha-\varepsilon}},[b, a]=a^{p^{\alpha-\gamma}}\right\rangle, \text { where } \alpha, \beta, \gamma, \varepsilon \in \square, \alpha-1 \gamma<2 \gamma, \alpha \leq \beta \\
& \quad \text { and } \alpha \leq \beta+\varepsilon .
\end{aligned}
$$

Moreover, metacyclic $p$-groups are also classified into two types, namely negative and positive type [12]. The following notations for these two types which are used in this paper are represented as follows:

$$
G(\alpha, \beta, \omega, \gamma, \pm) \cong\left\langle a, b: a^{p^{\alpha}}=1, b^{p^{\beta}}=a^{p^{\alpha-\varepsilon}},[b, a]=a^{t}\right\rangle \text {, where } \alpha, \beta, \gamma, \varepsilon \in \square, t=p^{\alpha-\gamma} \pm 1 .
$$

If $t=p^{\alpha-\gamma}-1$, then the group is called a metacyclic of negative type and it is of positive type if $t=p^{\alpha-\gamma}+1$. Thus, $G(\alpha, \beta, \varepsilon, \gamma,-)$ denotes the metacyclic group of negative type, while $G(\alpha, \beta, \varepsilon, \gamma,+)$ denotes the positive type. These two notations are shortened to $G(p,+)$ and $G(p,-)$, respectively ([11], [12]).

Since this paper focuses on metacyclic $p$-groups of positive type, thus the following theorem gives the presentations of the two groups of positive type.

\section{Theorem 1.1}

[12] Let $G$ be a metacyclic 2-group of positive type of nilpotency class of at least three. Then $G$ is isomorphic to one of the following types:

$$
\begin{aligned}
& \text { (1.1.1) } G \cong\left\langle a, b: a^{2^{\alpha}}=b^{2^{\beta}}=1,[b, a]=a^{2^{\alpha-\gamma}}\right\rangle, \alpha, \beta, \gamma \in \square, \beta \geq \gamma 1+\gamma<\alpha<2 \gamma . \\
& \text { (1.1.2) } G \cong\left\langle a, b: a^{2^{\alpha}}=1, b^{2^{\beta}}=a^{2^{\alpha-\varepsilon}},[b, a]=a^{2^{\alpha-\gamma}}\right\rangle, 1+\gamma<\alpha<2 \gamma, \gamma \leq \beta, \alpha \leq \beta+\varepsilon .
\end{aligned}
$$


The followings are some basic concepts of graph theory that are needed in this paper. These concepts can be found in one of the references ([13], [14]).

A graph $\Gamma$ is a mathematical structure consisting of two sets namely vertices and edges which are denoted by $V(\Gamma)$ and $E(\Gamma)$, respectively. The graph is called directed if its edges are identified with ordered pair of vertices. Otherwise, $\Gamma$ is called indirected. Two vertices are adjacent if they are linked by an edge. A complete graph is a graph where each ordered pair of distinct $n$ vertices are adjacent, denoted by $K_{n}$, while it is null if there is no vertices in $\Gamma$. The graph is called empty if there are no edges linked between its vertices. In this paper, $K_{e}$ denotes empty graph, while $K_{0}$ denotes null graph.

In 1990, a new graph called graph related to conjugacy class was introduced by Bertram et al. [15]. The vertices of this graph are non central conjugacy classes i.e $|V(\Gamma)|=K(G)-|Z(G)|$, where $K(G)$ is the number of conjugacy class of a group and $Z(G)$ is the center of a group $G$. A pair of vertices of this graph are connected by an edge if their cardinalities are not coprime.

In 2013, Omer et al. [16] extended the work on the conjugacy class graph by introducing a generalized conjugacy class graph whose vertices are non-central orbits under group action on a set.

This paper is structured as follows: Section 1 provides some fundamental concepts of group theory and graph theory which are used in this paper. In Section 2, we state some of previous works, which are related to the commutativity degree, in particular related to the probability that a group element fixes a set and conjugacy class graph. The main results are presented in Section 3.

\section{Preliminaries}

In this section, we provide some previous works related to the commutativity degree, more precisely to the probability that an element of a group fixes a set and graph theory, in particular to graph related to conjugacy class.

Recently, Omer et al. [7] extended the commutativity degree by defining the probability that an element of a group fixes a set of size two, given in the following.

\section{Definition 2.1}

[7] Let $G$ be a group. Let $S$ be a set of all subsets of commuting elements of $G$ of size two. If $G$ acts on $S$, then the probability of an element of a group fixes a set is defined as follows:

$$
P_{G}(S)=\frac{\{(g, s) \mid g s=s: g \in G, s \in S\} \mid}{|S||G|} .
$$




\section{Theorem 2.1}

[7] Let $G$ be a finite group and let $X$ be a set of elements of $G$ of size two in the form of $(a, b)$ where $a$ and $b$ commute. Let $S$ be the set of all subsets of commuting elements of $G$ of size two and $G$ acts on $S$. Then the probability that an element of a group fixes a set is given by

$$
P_{G}(S)=\frac{K(S)}{|S|} \text {, where } K(S) \text { is the number of orbits of } S \text { in } G \text {. }
$$

In 2014, Mustafa et al. [17] has extended the work in [7] by restricting the order of $\Omega$. The following theorem illustrates their results.

\section{Theorem 2.2}

[17] Let $G$ be a finite group and let $S$ be a set of elements of $G$ of size two in the form of $(a, b)$, where $a, b$ commute and $|a|=|b|=2$. Let $\Omega$ be the set of all subsets of commuting elements of $G$ of size two and $G$ acts on $\Omega$. Then the probability that an element of a group fixes a set is given by $P_{G}(\Omega)=\frac{K(\Omega)}{|\Omega|}$, where $K(\Omega)$ is the number of conjugacy classes of $\Omega$ in $G$.

Recently, Mustafa et al. [18] obtained the probability that an element of a group fixes a set for metacyclic 2-groups of negative type of nilpotency class two and class at least three. The probability is also computed under conjugate action for metacyclic 2-groups of positive type of nilpotency class at least three [19]. In addition, Mustafa et al. [20] found the probability that a group element fixes a set for semi-dihedral groups and quasi-dihedral groups.

In this paper, the probability that a positive type metacyclic 2-group element fixes a set is computed. Furthermore, the obtained results are then applied to graph theory by using the orbits that are obtained under group action on a set to graph conjugacy class.

Some related works on conjugacy class graph include Bianchi et al. [21] who studied the regularity of the graph related to conjugacy classes. In addition, Moreto et al. [22] in 2005 classified the groups in which conjugacy classes sizes are not coprime for any five distinct classes. Furthermore, You et al. [23] also classified the groups in which conjugacy classes are not set-wise relatively prime for any four distinct classes. Moreover, Moradipour et al. [24] used the graph related to conjugacy classes to find some graph properties of some finite metacyclic 2-groups.

Recently, Omer et al. [16] introduced generalized conjugacy class graph, given in the following definition.

\section{Definition 2.2}

[16] Let $G$ be a finite group and $\Omega$ a set of $G$. Let $A$ be the set of commuting element in $\Omega$, i.e $\{\omega \in \Omega: \omega g=g \omega, g \in G\}$. Then the generalized conjugacy class graph $\Gamma_{G}^{\Omega_{c}}$ is defined as a graph whose vertices are non-central orbits under group action on a set, 
that is $\left|V\left(\Gamma_{G}^{\Omega_{c}}\right)\right|=K(\Omega)-|A|$. Two vertices $\omega_{1}$ and $\omega_{2}$ in $\Gamma_{G}^{\Omega_{c}}$ are adjacent if their cardinalities are not coprime, i.e $\operatorname{gcd}\left(\omega_{1}, \omega_{2}\right) \neq 1$.

Moreover, the generalized conjugacy class graph is determined for the symmetric groups and alternating groups [25].

\section{Main Results}

This section provides our main results. First, the probability that an element of metacyclic 2-groups of positive type of nilpotency class at least three fixes a set is computed. Then the second part relates the obtained results to generalized conjugacy class graph.

Throughout this section, let $S$ be a set of elements of $G$ of size two in the form of $(a, b)$, where $a$ and $b$ commute and $|a|=|b|=2$. Let $\Omega$ be the set of all subsets of commuting elements of $G$ of size two and $G$ acts on $\Omega$ by regular action.

\section{The Probability That a Metacyclic 2-Group Element Fixes a Set}

\section{Theorem 3.1}

Let $G$ be a finite group of type (1.1.1), $G \cong\left\langle a, b: a^{2^{\alpha}}=b^{2^{\beta}}=1,[b, a]=a^{2^{\alpha-\gamma}}\right\rangle$, where $\alpha, \beta, \gamma \in \square, 1+\gamma<\alpha<2 \gamma, \beta \geq \gamma$. If $G$ acts regularly on $\Omega$, then $P_{G}(\Omega)=1$.

\section{Proof}

If $G$ acts regularly on $\Omega$, then there exists an element $g \in G$ such that $g \omega_{1}=\omega_{2}$ for $\omega_{1}, \omega_{2} \in \Omega$. The elements of order two in $G$ are $a^{2^{\alpha-1}}, b^{2^{\beta-1}}$ and $a^{2^{\alpha-1}} b^{2^{\beta-1}}$. Therefore, the elements of $\Omega$ are stated as follows: Two elements in the form of $\left(a^{2^{\alpha-1}}, a^{2^{\alpha-1} i} b^{2^{\beta-1}}\right), 0 \leq i \leq 2^{\alpha}$, and one element in the form $\left(b^{2^{\beta-1}}, a^{2^{\alpha-1}} b^{2^{\beta-1}}\right)$. Then $|\Omega|=3$ . By regular action, there exists an element $g \in G$ for $\omega_{1}, \omega_{2} \in \Omega$. such that $g \omega_{1}=\omega_{2}$. The orbits of $\Omega$ can be described as follows: One orbit in the form of $\left(a^{2^{\alpha-1}}, b^{2^{\beta-1}}\right)$, one orbit in the form of $\left(a^{2^{\alpha-1}}, a^{2^{\alpha-1}} b^{2^{\beta-1}}\right)$ and one orbit in the form of $\left(b^{2^{\beta-1}}, a^{2^{\alpha-1}} b^{2^{\beta-1}}\right)$. Thus there are three orbits. Using Theorem 2.2, then $P_{G}(\Omega)=1$.

\section{Theorem 3.2}

Let $G$ be group of type (1.1.2), $G \cong\left\langle a, b: a^{2^{\alpha}}=1, \quad b^{2^{\beta}}=a^{2^{\alpha-\varepsilon}},[b, a]=a^{2^{\alpha-\gamma}}\right\rangle$, $1+\gamma<\alpha<2 \gamma, \gamma \leq \beta, \alpha \leq \beta+\varepsilon$. If $G$ acts regularly on $\Omega$, then $P_{G}(\Omega)=1$. 


\section{Proof}

If $G$ acts regularly on $\Omega$, then there exists an element $g \in G$ such that $g \omega_{1}=\omega_{2}$ for $\omega_{1}, \omega_{2} \in \Omega . g \in G$. Thus, the elements of order two in $G$ are $a^{7 \times 2^{\alpha-4}+2^{\alpha-1}} b^{\beta^{\beta-1}}, \quad a^{7 \times 2^{\alpha-4}} b^{2^{\beta-1}}$ and $a^{2^{\alpha-1}}$. Hence, the elements of $\Omega$ are stated as follows: Two elements are in the form of $\left(a^{2^{\alpha-1}}, a^{7 \times 2^{\alpha-4}+2^{\alpha-1} i} b^{2^{\beta-1}}\right), 0 \leq i \leq 1$, and there is one element in $\Omega$ which are in the form of of $\left(a^{7 \times 2^{\alpha-4}} b^{2^{\beta-1}}, a^{7 \times 2^{\alpha-4}+2^{\alpha-1}} b^{2^{\beta-1}}\right)$, from which it follows that $|\Omega|=3$. By regular action, there exists an element $g \in G$ for $\omega_{1}, \omega_{2} \in \Omega$. such that $g \omega_{1}=\omega_{2}$. The orbits of $\Omega$ can be described as follows: One orbit in the form of $\left(a^{2^{\alpha-1}}, a^{7 \times 2^{\alpha-4}} b^{2^{\beta-1}}\right)$, one orbit in the form of $\left(a^{2^{\alpha-1}}, a^{7 \times 2^{\alpha-4}+2^{\alpha-1}} b^{2^{\beta-1}}\right)$ and one orbit in the form of $\left(a^{7 \times 2^{\alpha-4}} b^{2^{\beta-1}}, a^{7 \times 2^{\alpha-4}+2^{\alpha-1}} b^{2^{\beta-1}}\right)$. Thus there are three orbits. Using Theorem 2.2, then $P_{G}(\Omega)=1$.

\section{The Generalized Conjugacy Class Graph}

In this section, the results are related to generalized conjugacy class graph. First, the generalized conjugacy class graph of metacyclic 2-groups of positive type of nilpotency class of at least three is found, starting with the group of type (1.1.1).

\section{Theorem 3.3}

Let $G$ be a finite group of type (1.1.1), $G \cong\left\langle a, b: a^{2^{\alpha}}=b^{2^{\beta}}=1,[b, a]=a^{2^{\alpha-\gamma}}\right\rangle$, where $\alpha, \beta, \gamma \in \square, 1+\gamma<\alpha<2 \gamma, \beta \geq \gamma$. If $G$ acts regularly on $\Omega$, then $\Gamma_{G}^{\Omega_{c}}=K_{e}$.

\section{Proof}

Based on Theorem 3.1, there are three orbits all of size one. Using Definition 2.2, $\Gamma_{G}^{\Omega_{c}}$ consists of three isolated vertices, which are in the form of $\left(b^{2^{\beta-1}}, a^{2^{\alpha-1}} b^{2^{\beta-1}}\right)$, $\left(a^{2^{\alpha-1}}, a^{2^{\alpha-1}} b^{2^{\beta-1}}\right)$ and $\left(a^{2^{\alpha-1}}, b^{2^{\beta-1}}\right)$. The proof then follows.

\section{Theorem 3.4}

Let $G$ be group of type (1.1.2), $G \cong\left\langle a, b: a^{2^{\alpha}}=1, \quad b^{2^{\beta}}=a^{2^{\alpha-\varepsilon}},[b, a]=a^{2^{\alpha-\gamma}}\right\rangle$, $1+\gamma<\alpha<2 \gamma, \gamma \leq \beta, \alpha \leq \beta+\varepsilon$. If $G$ acts regularly on $\Omega$, then $\Gamma_{G}^{\Omega_{c}}=\left\{\begin{array}{l}K_{e}, \text { if } \alpha>\beta+1, \\ K_{0}, \text { if } \alpha \leq \beta+1 .\end{array}\right.$

\section{Proof}

In the first case, the proof is similar to the proof of Theorem 3.3. When $\alpha \leq \beta+1$ and based on Theorem 3.3 in [19] $P_{G}(\Omega)=1$ under conjugate action and by using 
Theorem 3.1 in [19] all commuting element of $G$ of order two are in the center of $G$. Thus, $K(\Omega)=|\Omega|=A$. Using the vertices adjacency of the generalized conjugacy class graph, $\Gamma_{G}^{\Omega_{c}}$ is null.

\section{Conclusion}

In this paper, the probability that a group element fixes a set under regular action is found for metacyclic 2-groups of positive type of nilpotency class of at least three. The results obtained are then applied to generalized conjugacy class graph.

\section{Acknowledgments}

The first author would like to acknowledge the Ministry of Higher Education in Libya for his $\mathrm{PhD}$ scholarship. The authors would also like to acknowledge Ministry of Education (MOE) Malaysia and Universiti Teknologi Malaysia for the financial funding through the Research University Grant (GUP) Vote No 08H07.

\section{References}

[1] Miller, G. A. 1944, "Relative Number of Non-invariant Operators in a Group," Proc. Nat. Acad. Sci. USA. 30(2): 25-28.

[2] Erdos, P., and Turan, P., 1968, "On Some Problems of a Statistical Group Theory," IV, Acta Math. Acad Sci. Hungaricae. 19: 413-435.

[3] Gustafson, W. H. 1973, "What is the Probability That Two Group Elements Commute?," The American Mathematical Monthly., 80(9):10311034.

[4] MacHale, D., 1974, "How Commutative Can a Non-Commutative Group Be?," The Mathematical Gazette, 58(405): 199-202.

[5] Sherman, G. J., 1975, "What is the Probability an Automorphsim Fixes a Group Element?," The American Mathematical Monthly. 82 (3): 261-264.

[6] Moghaddam, M. R. R., Saeedi, F., and Khamseh, E., 2011, "The Probability of an Automorphism Fixing a Subgroup Element of a Finite Group". Asian-European Journal of Mathematics. 4 (2): 301-308.

[7] Omer, S. M. S., Sarmin, N. H., Erfanian, A., and Moradipour, K., 2013, "The Probability That an Element of a Group Fixes a Set and the Group Acts on Set by Conjugation", International Journal of Applied Mathematics and Statistics, 32(2): 111-117.

[8] Rotman, J. J., 2002, “Advanced Modern Algebra,” New Jersey 07458: Pearson Education,Inc. Upper Saddle River. 
[9] Beardon, A. F., 2005, “Algebra and Geometry," Cambridge University Press.

[10] Goodman, F. M., 2003, "Algebra abstract and concrete .stressing symmetry," Person Education,Inc.Upper Saddle River.

[11] King, B. W., 1973, "Presentation of Metacyclic Groups," Bull Aust Math Soc, $103-131$.

[12] Beuerle, J. R., 2005, "An Elementary Classification of Finite Metacyclic p-Groups of Class at Least Three," Algebra Colloq., 12(4): 553-562.

[13] Bondy, J., and Murty, G., 1982, “Graph Theory With Application,” North Holand, Boston New York.

[14] Godsil, C., and Royle, G., 2001, “Algebraic Graph Theory,” Springer, Boston New York.

[15] Bertram, E. A., Herzog, M., and Mann, A., 1990, "On a Graph Related to Conjugacy Classes of Groups," Bull London Math Soc. 22: 569-575.

[16] Omer, S. M. S., Sarmin, N. H., and Erfanian, A., "Generalized Conjugacy Class Graph of Some Finite Non-abelian Groups," AIP Conf. Proc. In press.

[17] El-sanfaz, M. A., Sarmin, N. H., and Omer, S. M. S., 2014, "The Probability That an Element of the Dihedral Groups Fixes a Set," International Journal of Applied Mathematics and Statistics, 52(1): 1-6.

[18] El-sanfaz, M. A., Sarmin, N. H., and Omer, S. M. S., 2014, "The Probability that an Element of Metacyclic 2-Groups Fixes a Set," World Applied Sciences Journal, 32(3): 459-464.

[19] El-sanfaz, M. A., Sarmin, N. H., and Omer, S. M. S., 2014, "The Probability that an Element of Metacyclic 2-Groups of Positive Type Fixes a Set," Jurnal Teknologi, 71(1): 7-10.

[20] El-sanfaz, M. A., Sarmin, N. H., and Omer, S. M. S., 2015, “On The Probability That a Group Element Fixes a Set and Its Generalized Conjugacy Class Graph," International Journal of Mathematical Analysis. 9(4): 161-167.

[21] Bianchi, M., Chillag, D., Mauri, A., Herzog,M., and Scoppola, C., 1992, “ Applications of a Graph Related to Conjugacy Classes in Finite Groups," Arch Math., 58: 126-132.

[22] Moreto, A., Qian, G., and Shi, W., 2005, "Finite Groups Whose Conjugacy Class Graphs Have Few Vertices,” Arch. Math. 85: 101-107.

[23] You, X., Qian, G., and Shi, W., 2005, "A New Graph Related to Conjugacy Classes of Finite Groups," arXivmath0510015 [math.GR]. 
[24] Moradipour, K., Sarmin, N. H., and Erfanian, A., 2013, "On Graph Associated to Conjugacy Classes of Some Metacyclic 2-Groups," Journal of Basic and Applied Scientific Research. 3(1): 898-902.

[25] Omer, S. M. S., Sarmin, N. H., and Erfanian, A., 2013, "The Probability that an Element of a Symmetric Group Fixes a Set and Its Application in Graph Theory," World Applied Sciences Journal. 27(12):1637-1642. 